\title{
In silico self-assembly of nanoparticles with applications in drug delivery
}

\author{
Edithe Selwa and Bogdan I. Iorga \\ Institut de Chimie des Substances Naturelles, CNRS UPR 2301, Université \\ Paris-Saclay, Labex LERMIT, 1 avenue de la Terrasse, 91198 Gif-sur- \\ Yvette, France
}

\begin{abstract}
The use of nanoparticles for drug delivery applications has known an increasing success during the last years, together with the computational methods that are particularly useful for modeling the self-assembly process. The granularity at which particles are represented, force field parameters, initial setup and system composition are important factors influencing the outcome of simulations that have been the subject of intense research. In this chapter, the applications of these in silico approaches in drug delivery will be discussed, highlighting the importance of nanoparticle's composition: peptides, copolymers, lipids, or a mixture of them.
\end{abstract}

\section{Introduction}

During the recent years, nanotechnology has generated a great deal of interest throughout the biological sciences, due to its potential to provide novel approaches to therapeutic and diagnostic drug delivery. Traditional drug formulations are often associated with poor protection of the active compound from the biological environments and a lack of controlled release. ${ }^{1}$ In this chapter we will present briefly the computational methods that are currently

\section{RESERVE THIS SPACE}


used to investigate the self-assembly process, as well as the drug loading and release from nanoparticles. In the second part, representative in silico studies describing these processes will be presented, with a special emphasis on the techniques that are specific for each step and on the innovative approaches (multiscale simulations, doubly thermoresponsive nanoparticles, simultaneous delivery of multiple drugs, etc.). A more detailed presentation and discussion of some of these studies can be found in a recent review by Thota and Jiang. ${ }^{2}$

\section{Methods}

The systems that are to be studied can be represented at different levels of precision, depending on the size and the complexity of the system, the duration of the self-assembly process, etc.

Full-atom (FA) representations allow a description of the system with atomistic details. Among the all-atom force fields available, $\mathrm{CHARMM}^{3}$ and the associated CHARMM General Force Field $(\mathrm{CGenFF}){ }^{4}$ as well as $\mathrm{AMBER}^{5}$ and GAFF, ${ }^{6}$ are the most often employed for such molecular dynamics (MD) simulations, together with GROMOS, ${ }^{7}$ which is a typical united-atom force field. However, the processes involving self-assembly of nanoparticles, drug loading and release occur over a long-time scale, on the order of microseconds or longer. Therefore, these processes are relatively difficult to reproduce by allatom simulations, since these time-consuming approaches are computationally very expensive. However, recent advances in computation algorithms, especially in GPU-based computation, expanded significantly the scope of these simulations.

Coarse-grain (CG) models overcome these limitations by replacing fullatomistic details by lower resolution particles, while retaining most of the chemical specificity. MARTINI ${ }^{8-9}$ is one of the most used CG force field, which can well describe peptides, lipids, membranes, a wide variety of copolymers and other organic molecules. The main limitations of this force field are the rapid freezing and nucleation of water that may be observed at about $290 \mathrm{~K},{ }^{8}$ and the fact that transition of secondary structures cannot be accessed.

Dissipative Particle Dynamics (DPD) ${ }^{10-12}$ is a MD-based approach with higher level CG representation that is commonly applied to simulate drug loading and delivery in large nanoparticles. This technique simultaneously resolves both the molecular and continuum scales and reproduces the hydrodynamic behavior, to examine phase segregation in multicomponent lipid vesicles. ${ }^{13}$ In this way, the simulation length scales that are accessible are much larger than those by conventional MD. The size of dissipative particles can be freely tuned, but it cannot always be controlled with precision. The parameters are not transferable. 
Energy-preserving dissipative particle dynamics $\left(\right.$ eDPD) ${ }^{14}$ is an extension of the classical DPD method to non-isothermal fluid systems, predicting the diffusivity and viscosity consistent with experimental data. In this model, each particle's internal energy is explicitly modeled as a degree of freedom.

These methodologies can also be combined to represent the same system, either sequentially ${ }^{15}$ (CG or DPD representations are used for the self-assembly step, then converted into a FA representation for more detailed analysis) or simultaneously ${ }^{16}$ (multiscale models combining CG or DPD representations for solvent and a FA representation for solute).

\section{Applications}

\section{Peptide nanoparticles}

Some synthetic polymers possess cell toxicity and therefore are not useful for clinical applications. Ideal carriers for drug delivery must be non-toxic, nonimmunogenic, biocompatible, biodegradable and kinetically stable. The amphiphilic peptides possess most of these properties and are therefore increasingly used in drug delivery. ${ }^{2}$ By controlling the ratio of hydrophobic and hydrophilic residues in amphiphilic peptides, the peptide sequence or the concentration in solution, various morphologies such as vesicles, micelles, fibers, and hydrogels can be obtained by self-assembly. Typically, their sizes are below $100 \mathrm{~nm}$, small enough to avoid an immune reaction from the human body. $^{2,17}$

\section{Short peptides}

Extensive CGMD simulations of diphenylalanine (FF) peptides assembly showed the formation of nanovesicles and nanotubes as the main products, together with new ordered planar bilayers and a rich variety of other vesicle-like structures including toroid, ellipsoid, discoid, and pot-shaped vesicles (Figure 1). The assembly pathways are concentration-dependent, the fusion of small vesicles and bilayers being observed at low concentration, whereas at high concentration the formation of a bilayer seems to be favored. Subsequent allatom MD simulations on a fine-grained structure of a spherical vesicle from the CGMD trajectory shows that FF nanostructures are stabilized by a combination

of T-shaped aromatic stacking, interpeptide head-to-tail hydrogen-bonding, and peptide water hydrogen-bonding interactions. ${ }^{18}$ 


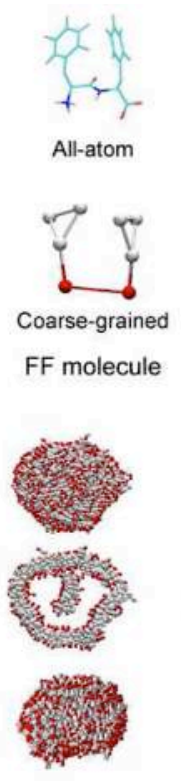

(c) pot-shaped
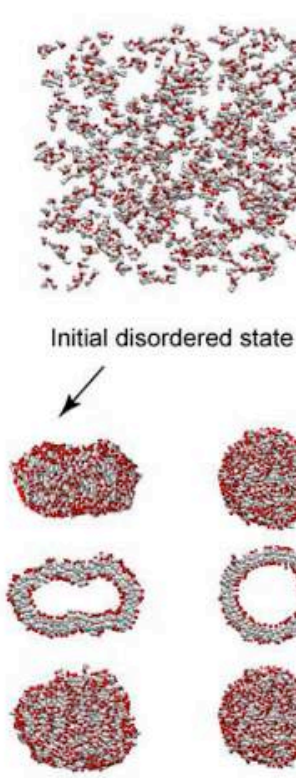

(d) discoid

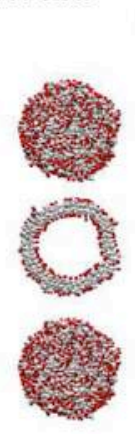

(e) sphere

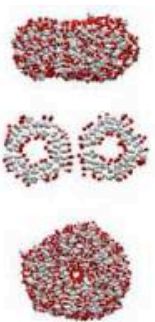

(a) toroid

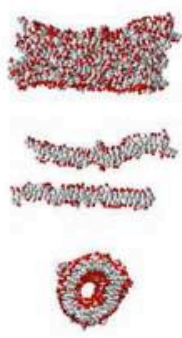

(f) nanotube

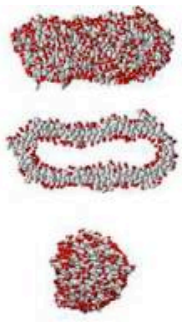

(b) ellipsoid

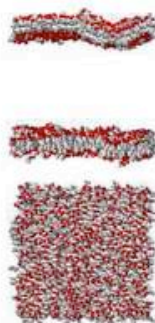

(g) bilayer

Figure 1. Initial disordered state and seven representative well-organized diphenylalanine peptide assemblies generated in MD simulations. For each nanostructure, a side view (top row), a cross-sectional view through its center of mass and the axis parallel to its principal axis (middle row), and a top view (bottom row) are shown. The main-chain beads are in red, and the side-chain beads are in white. There are three families of structures: vesicles with different shapes: (a) toroid, (b) ellipsoid, (c) pot-shaped, (d) discoid, and (e) spherical vesicles; nanotube (f); and bilayer (g). For clarity, a close-up of one dipeptide together with its atomistic structure is shown in the top-left corner. In the atomistic structure, carbon atoms are in cyan, oxygen atoms in red, hydrogen atom in white, and nitrogen atom in blue. Reproduced from reference ${ }^{18}$.

In a complementary study, all-atom MD simulations in explicit water were performed on both zwitterionic and capped (uncharged) versions of the FF peptide to understand the early stages of self-assembly. The amphiphilic nature of FF seems to be the key for understanding its self-assembly, and the early precursors to nanotube structures are likely to involve substantial hydrophobic clustering, rather than hexamer ring motifs. ${ }^{19}$ Similarly, detailed atomistic MD simulations of FF and dialanine (AA) peptides were carried out in two solvents, water and methanol. An important self-assembling propensity of FF in water was observed, while in methanol this propensity was very weak. For AA the 
tendencies are the same, but the differences between the two solvents are substantially smaller. ${ }^{20}$

Guo et al. described a study of triphenylalanine (FFF) peptides selfassembly using CGMD simulations, showing that solid nanometer-sized nanospheres and nanorods with substantial $\beta$-sheet content are formed. In contrast with the formation mechanism of water-filled FF nanovesicles and nanotubes, intermediate bilayers were not observed during the self-assembly process of FFF nanospheres and nanorods. ${ }^{21}$

The ability to predict the aggregation propensity (AP) of short peptides was evaluated by the screening of 400 dipeptide combinations using CGMD simulations, in order to determine the best precursors for self-assembly. Systems with the best scores were selected for longer simulations, and overall the results are in good agreement with data from literature. ${ }^{22}$ An extension of this work on a dataset containing 8,000 tripeptides indicated only a weak correlation between hydrophilicity and AP, confirming that self-assembly propensity is not simply a measure of hydrophobicity. The analysis of these results provided a set of rules for the design of tripeptides with improved self-assembly propensities: aromatic amino acids are most favorable in positions 2 and 3 in a tripeptide, and positive and hydrogen-bonding residues favor position 1 (N-terminus) and negative residues position 3 (C-terminus). Following these rules, a number of peptides were synthesized and characterized, allowing the identification of the first unprotected full-L tripeptides (KYF, KYY, KFF and KYW) able to form a hydrogel at neutral $\mathrm{pH}^{23}$

\section{Long peptides}

The self-assembly of medium length amphiphilic peptides with the generic structures $F_{m} D_{n}$ and $F_{m} K_{n}$ was investigated by $\mu$ s-scale CGMD simulations. The most interesting results were obtained with $\mathrm{F}_{3} \mathrm{~K}_{4}$ and $\mathrm{F}_{6} \mathrm{~K}_{8}$, which formed quasispherical micelles with distinct core/shell structure. This result suggests that the optimal ratio of hydrophobic/hydrophilic residues is $3 / 4$. Increasing this ratio led to elliptical aggregates, whereas decreasing this ratio resulted in smaller and less stable micelles. ${ }^{24}$

Three ionic peptides EAK16, containing the same 16 residues but different amino acid sequences, were studied by CGMD simulations in bulk solution or in the presence of a hydrophobic surface at different $\mathrm{pH}$ values. In bulk solution, two peptides showed aggregation into fibrillar structures at all $\mathrm{pH}$ ranges. The third one formed globular assemblies around the neutral $\mathrm{pH}$ and fibrillar aggregates in low and high $\mathrm{pH}$ conditions. However, on the hydrophobic surface, this last peptide formed disc-shaped assemblies under neutral $\mathrm{pH}$ conditions. ${ }^{25}$ 
CGMD simulations were used to explore the self-assembling behavior of a FA32 amphiphilic peptide, $(\mathrm{AF})_{6} \mathrm{H}_{5} \mathrm{~K}_{15}$. The formation of micelles is observed, with Ala and Phe residues in the hydrophobic core, Lys in the hydrophilic shell, and amphiphilic His at the interface. The self-assembly is a three-step process, involving the formation of small clusters, their aggregation and merging into large clusters, and finally the formation of micelles. Higher peptide concentrations induces the formation of bigger micelles and a change in their shape, from quasi-spherical into elongated forms. ${ }^{26} \mathrm{~A}$ follow-up study from the same group provided more detailed information about the role of hydrophilic and hydrophobic residues in the self-assembly of FA32-like peptides. The selfassembly capability is reduced by increasing hydrophilicity, with the formation of smaller micelles or the presence of individual peptide chains, whereas increasing hydrophobicity leads to an increase in micelle size and to a morphology shift from spherical to fiber-like. ${ }^{27}$

The loading and the release of ibuprofen (IBU) in FA32 and its derivatives $\mathrm{F}_{12} \mathrm{H}_{5} \mathrm{~K}_{15}$ and $\mathrm{F}_{16} \mathrm{H}_{5} \mathrm{~K}_{15}$ was studied using CGMD simulations. IBU loading in FA32 led to quasi-spherical core/shell structured micelles, where IBU was predominantly located at the core along with Phe and Ala, while Lys and His were in the shell and at the core/shell interface, respectively. After loading, due to increased hydrophobic interactions, the micelles become larger, proportionally with the increase in the ratio drug/peptide. The morphology of IBU-loaded nanoparticles depends on the type of peptide and has a significant effect on IBU release profile. In FA32 derivatives, IBU loading led to different morphologies, a well-structured nanofiber being formed in $\mathrm{F}_{16} \mathrm{H}_{5} \mathrm{~K}_{15}$. IBU release from FA32 micelles upon $\mathrm{pH}$ change is slower than from $\mathrm{F}_{16} \mathrm{H}_{5} \mathrm{~K}_{15}$ nanofiber, suggesting that the former might be more appropriate for the controlled release. ${ }^{28}$

The amphiphilic self-assembling decapeptide 2 (SA2 peptide, sequence AcAAVVLLLWEE-COOH) was shown to spontaneously self-organize into hollow spheres. CGMD simulations were used to get more insight into the supramolecular structure of SA2 nanocarriers and the intermediates involved in the self-assembly process. The coarse-grained system was then converted into an atomistic representation, with a system containing 41 millions atoms. Subsequent FAMD simulations provided a more accurate knowledge on the atomic level geometry and dynamics of assembled peptides in relation to the supramolecular organization of the SA2 nanocarrier. It was shown that this organization involves interdigitated antiparallel $\beta$-sheets and not the liposomelike tail-to-tail orientation that is generally observed for the amphiphilic molecules. $^{15}$

MD simulations were carried out to provide insight into the effect of secondary structure on the self-assembly of $\beta$-peptides using hydrophobic blocks (composed of 10-mer or 20-mer of $\beta$-valine residues) and hydrophilic blocks (composed of 10-mer or 20-mer of positively charged 2-aminomethyl- 
3,3-dimethyl $\beta$-amino acid residues). In a good agreement with experimental data, all-atom free energy calculations showed that the association of a pair of homochiral hydrophobic blocks promotes self-assembly compared to the same hydrophobic blocks in a heterochiral form. CGMD simulations of mixtures containing hydrophobic and hydrophilic blocks showed that these molecules spontaneously form spherical micelles. ${ }^{29}$

A mixture of 15 and 23-residue branched, amphiphilic peptides that mimic phosphoglycerides in molecular architecture was shown to produce supramolecular self-assembly and form solvent-filled vesicles with 50-200 nm diameters. In contrast, CGMD simulations of the same system produced a welldefined bilayer. ${ }^{30}$ Further theoretical investigations would be needed, using bigger systems and possibly different peptide/water ratio, to determine the optimal conditions for obtaining an in silico model of the vesicles.

Atomistic MD simulations of an amphiphilic peptide with the sequence SLSLAAAEIKVAV ${ }^{31}$ and two simplified variants $\left(\mathrm{V}_{2} \mathrm{~A}_{4} \mathrm{E}_{3} \text { and } \mathrm{V}_{4} \mathrm{~A}_{2} \mathrm{E}_{3}\right)^{32}$ showed a self-assembly process resulting in the formation of cylindrical micelles and nanofibers. In a subsequent study, the self-assembly of the same peptide was investigated using CGMD simulations. A three-step mechanism was evidenced in the formation of nanofibers: i) formation of spherical micelles, ii) their aggregation into a three-dimensional network, and finally iii) the formation of a cylindrical nanofiber (Figure 2). ${ }^{33}$ A careful analysis of the free-energy landscape for this process showed the formation of a pillar-like intermediate state before the final cylindrical semifiber structure. Additionally, the head group assembly was found to occur on a much shorter time scale than tail group assembly. Although CGMD simulations might not be precise enough to draw such conclusions, it was proposed that the driving force leading to the formation of the micelle structure is enthalpic, originating from several factors, including the conformational energy of amphiphilic peptides and the electrostatic and van der Waals interaction energy between solvent molecules and between solvent and peptides. ${ }^{34-35}$

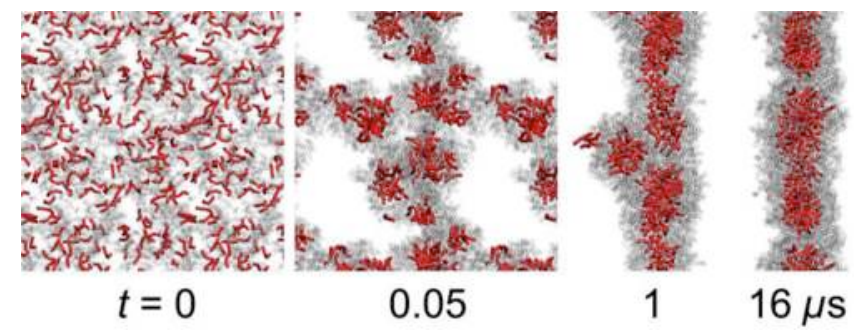

Figure 2. Coarse-grained molecular dynamics simulations showing selfassembly of peptide amphiphiles into spherical micelles, a three-dimensional network of micelles, and finally a cylindrical micelle fiber. Reproduced from reference ${ }^{33}$. 


\section{Amphiphilic copolymer nanoparticles}

Amphiphilic polymeric nanostructures have long been well-recognized as an excellent candidate for drug delivery applications. With the recent advances in the "top-down" and "bottom-up" approaches, development of well-defined polymeric nanostructures of different shapes has been possible. ${ }^{36}$ Under appropriate conditions, the amphiphilic copolymers can assemble into a variety of morphologies (spheres, cylinders, bicontinuous structures, lamellae, vesicles, micelles, fibers, and many other complex or hierarchical assemblies). These morphologies may be affected by polymer concentration, hydrophobic/hydrophilic ratio, end-group, and solvent content.

An interesting multiscale (dual resolution) approach that combines atomistic (GROMOS96) and coarse-grain (MARTINI) force fields was used to simulate the drug-polymer nanoparticle assembly by nano-precipitation from mixed solvents. The interaction between three poly(glycerol adipate) (PGA) polymer variants and the anticancer drug dexamethasone phosphate (DXMP) was studied (Figure 3), and the relative encapsulation efficiency and drug loading retrieved for the PGA polymers were in agreement with the experimental data. The water and acetone co-solvents were modeled as purely coarse-grained entities, whereas PGA and DXMP were modeled at both atomistic and coarse-grained levels, using virtual sites. In this way, all solutesolute interactions were treated fully atomistically, and the solute-solvent and solvent-solvent interactions were treated at the coarse-grain level. The simulations results showed that a balance between hydrophobic and hydrophilic moieties in the polymer is important for optimal drug incorporation. The C18 chains are required to interact favorably with DXMP, but having too many $\mathrm{C} 18$ chains decreases solubility, preventing the polymer from interacting with surrounding DXMP molecules. ${ }^{16}$ 

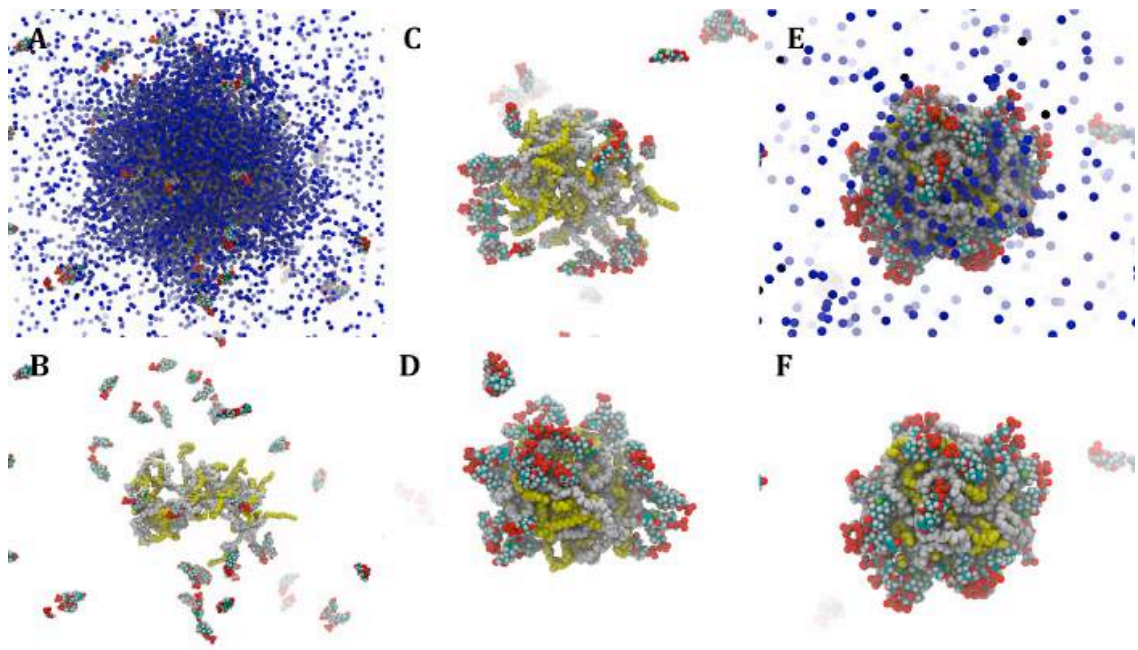

Figure 3. Snapshots taken during the 80 ns multiscale simulation of PGA20-coC18PGA80 with DXMP: (A) $5 \mathrm{~ns}$ (with acetone shown), (B) $5 \mathrm{~ns}$, (C) $30 \mathrm{~ns}$, (D) $50 \mathrm{~ns}$, (E) $80 \mathrm{~ns}$ (with acetone shown), and (F) $80 \mathrm{ns.}$ DXMP (multi), PGA (white with C18 chains yellow), acetone (blue). For clarity, water and coarsegrain virtual sites are not shown. Reproduced from reference ${ }^{16}$.

CGMD simulations were used to explore the self-assembly process of polyamidoamine (PAMAM) amphiphilic dendrimers (ADs) with different alkyl chains and generations, and the interaction of the resulting aggregates with siRNA. These simulations evidenced that hydrophobic tail length and hydrophilic head size, or dendron generation, have a critical impact on the size, shape, and self-organization of the aggregates, as well as on interactions with nucleic acids. ${ }^{37}$

A method, based on all-atom MD simulations using the AMBER/GAFF force field, ${ }^{5-6}$ was developed for the calculation of Flory-Huggins interaction parameters as a measure for the loading capability (solubility) of lipophilic drugs into polymer micelles. Very good agreement with experimental data was observed for four different drugs with significantly different lipophilicities. ${ }^{38}$

In a similar work, FAMD simulations allowed the prediction of solubilities for two drugs, fenofibrate and nimodipine, in a series of micelle-forming poly(ethylene oxide)- $b$-poly( $\varepsilon$-caprolactone) (PEO- $b$-PCL) block copolymers with combinations of blocks having different molecular weights. The results were in a good agreement with experimental data. ${ }^{39}$ The same protocol applied for two water insoluble anticancer drugs, cucurbitacin $\mathrm{B}(\mathrm{CuB})$ and cucurbitacin I (CuI), showed that in both cases the drug affinity increases with increasing PCL/PEO ratio. ${ }^{40}$ When the branched PEO-b-3PCL block copolymer was used, the solubility of cucurbitacin drugs $\mathrm{CuB}$ and $\mathrm{CuI}$ increased considerably 
compared to PEO- $b$-PCL, whereas the opposite behavior was observed in the case of the fenofibrate and nimodipine molecules. ${ }^{41}$

Diffusion of the hydrophobic drug $\mathrm{CuB}$ in linear PEO- $b$-PCL and branched PEO- $b-3$ PCL was studied using FAMD simulations in conjunction with different amounts of water. It was found that the drug diffusivity is insensitive to the water amount and double in linear PEO- $b$-PCL compared with those in the branched PEO- $b-3 \mathrm{PCL}$, due to a cumulative effect of stronger hydrogen bonding and lower swelling. ${ }^{42}$

Subtle structural characteristics (size, flexibility, rigidity) play a significant role in the efficiency of drug loading. A computational model to predict the affinity between the drug and its nanoparticulate carrier, e.g. DTO-SA $/ 5 \mathrm{~K}$ nanospheres, was developed by combining FAMD simulations with docking calculations. The model was able to successfully rank order three drugs (nutraceutical curcumin, anti-cancer drug paclitaxel and pre-hormone vitamin D3) in terms of experimentally observed drug loading at maximum into the nanospheres, by comparing it with computationally calculated binding energies. ${ }^{43}$

DPD simulations were used to get more insight into the structural organization and properties of pure paclitaxel and paclitaxel-loaded PEO- $b$-PLA systems. Alone, paclitaxel molecules formed ribbon or fiber-like structures in water. With the addition of the copolymer block, paclitaxel acted as a template and polymer molecules assembled around the paclitaxel structure to form core/shell structured fibers having a paclitaxel/PLA core and a PEO shell. ${ }^{44}$ DPD simulations were also carried out by the same group to study drug diffusion abilities into the core of micelles after the core-shell structure of the micelle is formed, which is the main issue affecting the drug loading efficiency. Important parameters such as topological structure of the drug, the hydrophobic block length of the polymer, and the compatibility between the drug and the hydrophobic block were shown to have significant effects on drug loading efficiencies and drug distributions inside micelles. ${ }^{45}$ Similar results were obtained from the investigation of doxorubicin (DOX) loading and $\mathrm{pH}$ controlled release from $\mathrm{pH}$-sensitive 4AS-PCL- $b$-PDEAE-MA- $b$-PPEGMA ${ }^{46}$ and $(\mathrm{PCL})_{2}(\mathrm{PDEA}-b \text {-PPEGMA })_{2}{ }^{47-48}$ micelles. More advanced in silico studies tried to rationalize the relationship between the structure of $\mathrm{pH}$-sensitive polymers and drug release behaviors from the micelles, showing that the "fireworks-like" three layer structure is very important for the drug release, and different drug distributions (e.g. i) in the $\mathrm{pH}$-sensitive layer, ii) both in the $\mathrm{pH}$ sensitive layer and in the core, or iii) in the core of micelles) generally induce different drug release processes. ${ }^{49}$

In some cases, a loading-dependent morphology transition can be observed, suggesting the maximum payload that the considered copolymer nanocarrier can accommodate under the selected conditions. In a representative study, the selfassembly of (D-L)-PLA/PEO di/triblock copolymers in aqueous environment 
and in the presence of a model drug (nifedipine) was investigated, based on a hybrid molecular simulation recipe. Atomistic MD simulations were used to obtain DPD input parameters, and this mesoscale technique was employed to derive the entire phase diagrams for these systems. It was observed that spherical aggregates are formed at low drug concentrations, which rearrange their assembly into cylindrical or eventually membrane-like aggregate as the payload amount increases. ${ }^{50}$

In another multiscale in silico study, FAMD simulations were used to estimate the Flory-Huggins interaction parameters and miscibility of binary components from a system representing the anti-cancer drug camptothecin (CPT) in a pH-sensitive amphiphilic copolymer, composed of hydrophobic poly( $\beta$-amino ester) (PAE) and hydrophilic methyl ether-capped poly(ethylene glycol) (PEG). DPD simulations were then employed to examine the micellization of PAE-PEG, CPT loading in PAE-PEG, and CPT release upon protonation from micelles/vesicles by a swelling-demicellization-releasing mechanism. ${ }^{51}$

An interesting multiscale approach was used to investigate the selfassembly of telodendrimer-based nanocarriers for anticancer drug delivery. CGMD simulations were carried out to represent the self-assembly process, followed by reverse mapping of the coarse-grained system to atomistic representation for structural analysis. This procedure provided important parameters like average size, drug-telodendrimer micellar stoichiometry, optimal drug loading capacity, and atomistic details such hydrogen-bonding and solvent accessible area of the nanocarrier. The computed results were in good agreement with the experimental data. ${ }^{52}$

Recent findings showed that the nanoparticle shape can be an important factor for modulating the efficacy of drug delivery process. Flexible, worm-like nanocarriers (Worms) increase the amount of drug delivered to tumors and shrink the tumors more effectively than spherical micelles (Spheres). A rational coarse grain ( $\mathrm{rCG}$ ) model based on all-atom MD simulations was built and used to investigate the shape-dependent effects in the delivery of the anticancer drug Taxol by block copolymer micelles. In this way, it was evidenced that twice as much drug loads into Worms than Spheres, fully consistent with experiments. ${ }^{53}$

The mechanism of transportation and delivery of hydrophilic drug components such as anticancer doxorubicin and mitoxantrone are of highest importance and poorly understood. CGMD simulations were employed to study how "patchy" polymeric micelle can assist the transportation of a hydrophilic drug across a lipid membrane and deliver it inside a biological lipid vesicle. Extensive simulations demonstrated successful encapsulation, transportation of the hydrophilic drug by "patchy" polymeric micelles and the release of a significant portion of drug inside the lipid vesicle (Figure 4). ${ }^{54}$ 


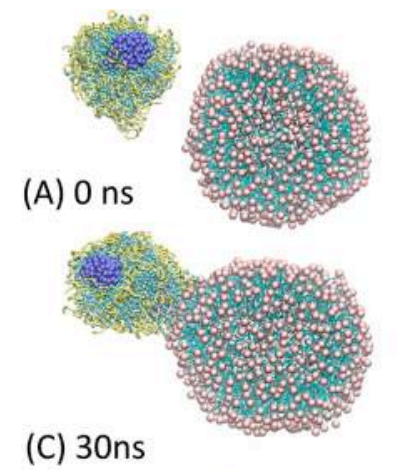

(B) $16 \mathrm{~ns}$

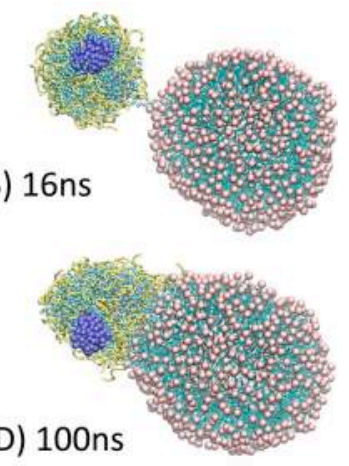

(E) 200ns

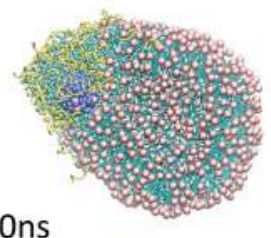

(F) 350ns

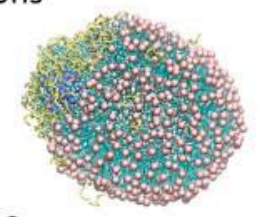

(G) 400ns

(D) $100 \mathrm{~ns}$

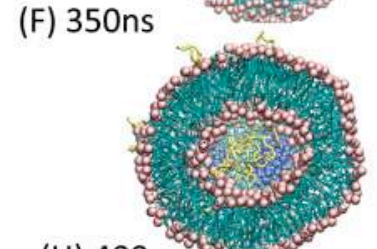

(H) 400ns

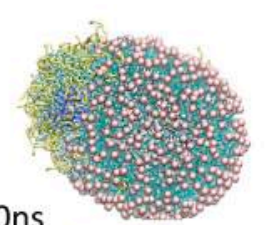

Figure 4. CGMD simulation snapshots of a binary $A B+C B$ polymeric micelle interaction with DPPC lipid vesicle are shown. Polymeric patches in the micelle are shown in blue along with hydrophobic (cyan) and hydrophilic (yellow) polymers. One of the CG beads in the lipid headgroup is shown in bead representation, while the rest of the lipid is drawn in stick representation. Water is not shown for clarity. The initially separated polymeric micelle (at $0 \mathrm{~ns}$ ) gradually penetrates the lipid vesicle (400 ns). The cross-sectional view of the final snapshot is shown in part H. Reproduced from reference ${ }^{54}$.

The interaction of Pluronic L64 block copolymer micelles with DPPC lipid bilayers was investigated using CGMD simulations showing, in agreement with experimental data, the release of triblock chains from the micelle inside the bilayer. The presence of a drug molecule inside the hydrophobic core of the micelle has a strong influence on this process, the micelle stability being the result of a complex interplay of drug-core and block-co-polymer-bilayer interactions modulating the structures of both the micelle and the bilayer. ${ }^{55}$

A recent study investigated the behavior of doubly thermoresponsive micelles and vesicles through computer simulations, using the recentlydeveloped eDPD methodology. ${ }^{14}$ Mesoscopic simulations were used to simulate 
the thermally induced self-assembly of LCST-UCST diblock copolymers, process triggered by the evolution of temperature over time and space. Both external and intrinsic factors can influence the assembly pathway of thermoresponsive micelles and thus determining the final morphology of the aggregate. It was found that thermoresponsive unilamellar vesicles can sustain repeated heating-cooling cycles in a thermal loading test, and the collapse probability and half-life of the vesicles under frequencies that cause vesicle destruction were quantified. The thermoresponsive bilayer membranes exhibit a very high ratio of molecular migration between the two leaflets during thermally induced inversion, process dominated by two molecular movement modes, flip and slip (Figure 5). ${ }^{56}$

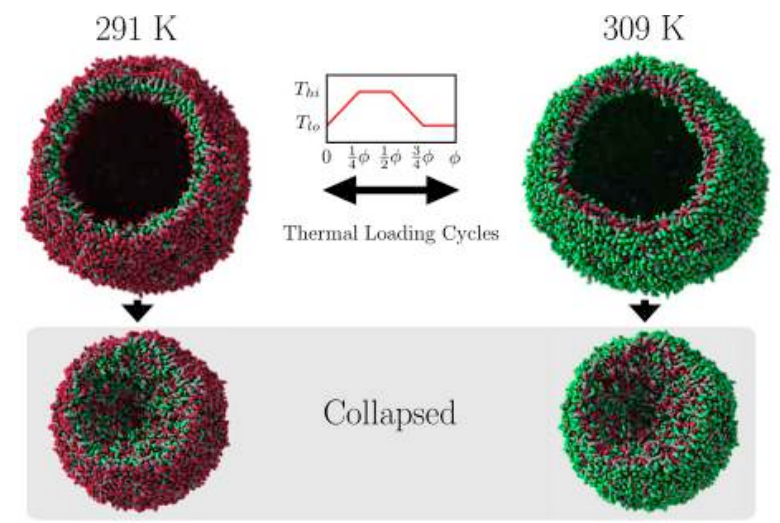

Figure 5. Thermoresponsive vesicles invert by diffusion and respond differently to various thermal loading frequencies. A vesicle formed by L2N5U2

thermoresponsive block copolymer can invert repeatedly when subjected to thermal loading cycles and may collapse irreversibly. Adapted from reference 56.

\section{Lipid nanoparticles}

Implicit solvent CGMD simulations were used to investigate the selfassembly and phase segregation of stable single and binary vesicles composed of two-tail phospholipids. It was shown that the degree of dissimilarity between the tail groups of the lipid species composing the vesicle can be used to tune the phase segregation in the vesicle bilayer. Accordingly, the interfacial tension increases with the difference between the tail groups, driving the phase segregation process to minimize the interfacial tension. ${ }^{13}$

A coarse-grained DPPC liposome model was loaded with varying amounts of hypericin, a natural compound proposed for use in photodynamic therapy. In 
agreement with experimental data, CGMD simulations performed on the microsecond time scale of liposomes with varying hypericin concentration showed that the liposome expands as the amount of hypericin is increased. These simulations also provided valuable information about the distribution of hypericins, their orientations within the lipid bilayer, and the potential of mean force for transferring a hypericin molecule from the interior aqueous "droplet" through the liposome bilayer (Figure 6). ${ }^{57}$
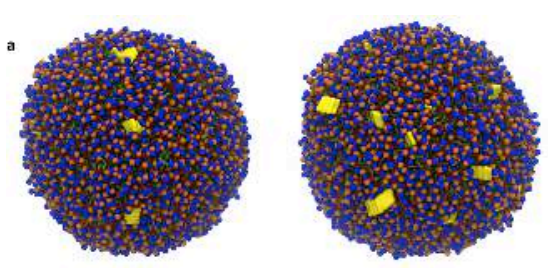

21 hypericin

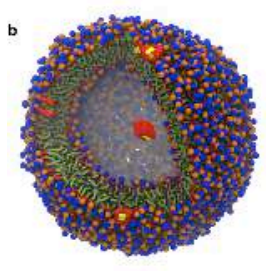

21 nypericin

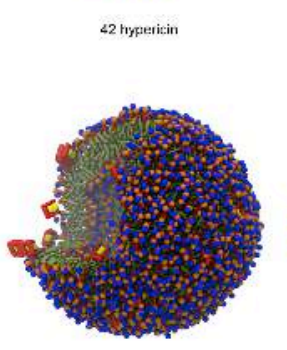

42 whericin

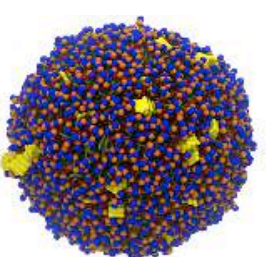

84 typesicin

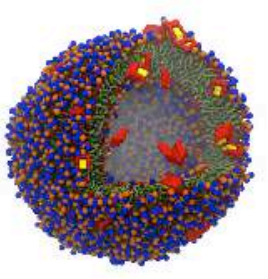

s4 hyperion

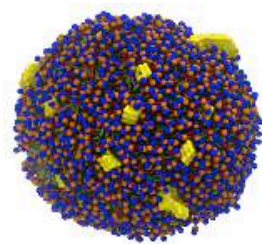

a4 hypericin

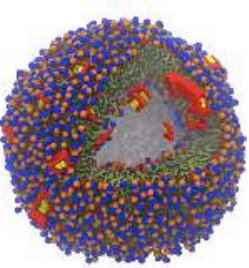

84 mycerici

Figure 6. (a) Final snapshots from the $10 \mu$ s molecular dynamics simulations of liposomes (red and green beads correspond to hydrophilic entities and gray beads to hydrophobic ones) loaded with hypericin molecules (in yellow). (b) Snapshots with some lipids removed in order to illustrate the binding of hypericin; the hydrophilic parts of hypericin are shown in red and the hydrophobic parts in yellow. Reproduced from reference ${ }^{57}$.

\section{Hybrid nanoparticles}

Using a novel coarse-grained peptide/polymer model, the spontaneous selfassembly process of a hybrid, lipid-like peptide amphiphile (palmitoyl- $\mathrm{V}_{3} \mathrm{~A}_{3} \mathrm{E}_{3}$ ) was studied using large-scale CGMD simulations. The formation of nanostructures of various sizes and shapes is observed as a function of the electrostatics and temperature. At optimal conditions, the self-assembly process starts by a quick micellization whose driving force is the hydrophobic interactions between alkyl tails, followed by a slow ordering process of neighboring peptide residues within a micelle and the formation of $\beta$-sheets exposing the hydrophobic core. Finally, spherical micelles merge together 
through an end-to-end mechanism to form cylindrical nanofibers. ${ }^{58}$ The assembly process was found to occur through multiple steps with transient intermediates that ultimately leads to the stabilized nanostructures including open networks of $\beta$-sheets, cylindrical nanofibers, and elongated micelles. ${ }^{59}$ Further work showed the important role of an increased hydrophobicity, which favors the progression from open networks of secondary structures toward closed cylindrical nanostructures containing either $\beta$-sheets or random coils. ${ }^{60}$

DPD simulations are generally the most appropriate tools for studying the loading and release of drugs from nanoparticles. In a representative study, micelles self-assembled from cholesterol-conjugated $\mathrm{His}_{10} \mathrm{Arg}_{10}$ (HR20-Chol) were used for the encapsulation of doxorubicin (DOX) at different $\mathrm{pH}$ conditions. Stronger DOX loading ability was observed at $\mathrm{pH}>6.0$, due to the hydrophobicity of histidine residues. At $\mathrm{pH}<6.0$, the dense structures of micelles adopted swollen conformations, thus facilitating the release of DOX from the core of micelles. ${ }^{61}$ Further atomistic simulations were performed to study the compatibility between each pair of components and to calculate the FloryHuggins interaction parameters between water, DOX, and other components in this system. ${ }^{62}$ A similar study was carried out later using doxorubicin-loaded micelles prepared from block polymers His(x)Lys10 $(\mathrm{x}=0,5,10)$ conjugated with docosahexaenoic acid. ${ }^{63}$

The nanoparticles can accommodate simultaneously several drugs and release them specifically in different experimental conditions, as shown in a recent study in which a dual-drug-loaded micelle was designed and constructed by self-assembly from a mixture of poly(propylene oxide)- $b$-poly $(\gamma$-benzyl-Lglutamate)- $b$-poly(ethylene glycol) (PPO- $b$-PBLG- $b$-PEG) triblock terpolymers and two model drugs, doxorubicin (DOX) and naproxen (Nap). In the micelles, the DOX is covalently linked to PBLG through an acid-cleavable hydrazone bond, whereas the Nap is physically encapsulated in the cores. DPD simulations were used for studying the drug loading and releasing behaviors of these micelles (with one or two drugs loaded simultaneously), which revealed that the micelles possess a core-shell-corona structure in which the PPO/Nap, PBLG/DOX, and PEG aggregate to form the core, shell, and corona, respectively. The release of DOX and Nap is $\mathrm{pH}$ - and thermosensitive and these dual-drug-loaded micelles were shown to be biocompatible at normal physiological conditions. ${ }^{64}$

In an elegant study, DPD simulations were used to investigate the selfassembly process of core-polyethylene glycol-lipid shell (CPLS) nanoparticles and their potential as drug delivery vehicles. The spherical core was functionalized on surface by PEG chains with the free ends covalently bonded with lipid molecules (lipid heads). The simulations showed the formation of a complete lipid bilayer shell at the surface of the PEGylated particle core upon addition of free lipids, leading to the formation of a CPLS nanoparticle. In order to evaluate the potential of CPLS nanoparticles as drug carriers, the 
internalization of nanoparticles (unloaded or loaded with hydrophilic molecules) into liposomes (lipid vesicles) was studied through DPD simulations. During this process, the rearrangement of the lipid bilayer around nanoparticles was evidenced, and this represents the most important step for the nanoparticle internalization via endocytosis into diseased cells. Overall, this work highlights the significant potential of CPLS nanoparticles as drug carriers with high efficacy and non-toxicity, as their surface chemistry is identical to liposome yet their stability is superior. ${ }^{65}$

\section{Acknowledgments}

Our laboratory is a member of the Laboratory of Excellence in Research on Medication and Innovative Therapeutics (LERMIT) supported by a grant from the French National Research Agency (ANR-10-LABX-33).

\section{References}

1. Pearson, R. T.; Avila-Olias, M.; Joseph, A. S.; Nyberg, S.; Battaglia, G., Smart polymersomes: Formation, characterization and applications. In Smart Materials for Drug Delivery, Vol. 1, Alvarez-Lorenzo, C.; Concheiro, A., Eds. Royal Society of Chemistry: Cambridge, UK, 2013; pp 179-207.

2. Thota, N.; Jiang, J., Computational amphiphilic materials for drug delivery. Front. Mater. 2015, 2, 64.

3. Brooks, B. R.; Brooks, C. L., 3rd; Mackerell, A. D., Jr.; Nilsson, L.; Petrella, R. J.; Roux, B.; Won, Y.; Archontis, G.; Bartels, C.; Boresch, S.; Caflisch, A.; Caves, L.; Cui, Q.; Dinner, A. R.; Feig, M.; Fischer, S.; Gao, J.; Hodoscek, M.; Im, W.; Kuczera, K.; Lazaridis, T.; Ma, J.; Ovchinnikov, V.; Paci, E.; Pastor, R. W.; Post, C. B.; Pu, J. Z.; Schaefer, M.; Tidor, B.; Venable, R. M.; Woodcock, H. L.; Wu, X.; Yang, W.; York, D. M.; Karplus, M., CHARMM: The biomolecular simulation program. J. Comput. Chem. 2009, 30, 1545-1614.

4. Vanommeslaeghe, K.; Hatcher, E.; Acharya, C.; Kundu, S.; Zhong, S.; Shim, J.; Darian, E.; Guvench, O.; Lopes, P.; Vorobyov, I.; Mackerell, A. D., Jr., CHARMM general force field: A force field for drug-like molecules compatible with the CHARMM all-atom additive biological force fields. $J$. Comput. Chem. 2010, 31, 671-690.

5. Case, D. A.; Cheatham, T. E., 3rd; Darden, T.; Gohlke, H.; Luo, R.; Merz, K. M., Jr.; Onufriev, A.; Simmerling, C.; Wang, B.; Woods, R. J., The Amber biomolecular simulation programs. J. Comput. Chem. 2005, 26, 1668-1688. 
6. Wang, J.; Wolf, R. M.; Caldwell, J. W.; Kollman, P. A.; Case, D. A., Development and testing of a general Amber force field. J. Comput. Chem. 2004, 25, 1157-1174.

7. Scott, W. R. P.; Hünenberger, P. H.; Tironi, I. G.; Mark, A. E.; Billeter, S. R.; Fennen, J.; Torda, A. E.; Huber, T.; Krüger, P.; van Gunsteren, W. F., The GROMOS biomolecular simulation program package. J. Phys. Chem. A 1999, 103, 3596-3607.

8. Marrink, S. J.; De Vries, A. H.; Mark, A. E., Coarse grained model for semiquantitative lipid simulations. J. Phys. Chem. B 2004, 108, 750-760.

9. Marrink, S. J.; Risselada, H. J.; Yefimov, S.; Tieleman, D. P.; De Vries, A. H., The MARTINI force field: Coarse grained model for biomolecular simulations. J. Phys. Chem. B 2007, 111, 7812-7824.

10. Hoogerbrugge, P.; Koelman, J., Simulating microscopic hydrodynamic phenomena with dissipative particle dynamics. EPL (Europhys. Lett.) 1992, $19,155$.

11. Koelman, J.; Hoogerbrugge, P., Dynamic simulations of hard-sphere suspensions under steady shear. EPL (Europhys. Lett.) 1993, 21, 363.

12. Espanol, P.; Warren, P., Statistical mechanics of dissipative particle dynamics. EPL (Europhys. Lett.) 1995, 30, 191.

13. Aydin, F.; Dutt, M., Bioinspired vesicles encompassing two-tail phospholipids: Self-assembly and phase segregation via implicit solvent coarse-grained molecular dynamics. J. Phys. Chem. B 2014, 118, 86148623.

14. Li, Z.; Tang, Y.-H.; Lei, H.; Caswell, B.; Karniadakis, G. E., Energyconserving dissipative particle dynamics with temperature-dependent properties. J. Comput. Phys. 2014, 265, 113-127.

15. Rad-Malekshahi, M.; Visscher, K. M.; Rodrigues, J. P.; de Vries, R.; Hennink, W. E.; Baldus, M.; Bonvin, A. M.; Mastrobattista, E.; Weingarth, M., The supramolecular organization of a peptide-based nanocarrier at high molecular detail. J. Am. Chem. Soc. 2015, 137, 7775-7784.

16. Mackenzie, R.; Booth, J.; Alexander, C.; Garnett, M.; Laughton, C. A., Multiscale modeling of drug-polymer nanoparticle assembly identifies parameters influencing drug encapsulation efficiency. J. Chem. Theory Comput. 2015, 11, 2705-2713.

17. Sun, L.; Zheng, C.; Webster, T. J., Self-assembled peptide nanomaterials for biomedical applications: Promises and pitfalls. Int. J. Nanomed. 2017, $12,73-86$.

18. Guo, C.; Luo, Y.; Zhou, R.; Wei, G., Probing the self-assembly mechanism of diphenylalanine-based peptide nanovesicles and nanotubes. ACS Nano 2012, 6, 3907-3918.

19. Jeon, J.; Mills, C. E.; Shell, M. S., Molecular insights into diphenylalanine nanotube assembly: All-atom simulations of oligomerization. J. Phys. Chem. B 2013, 117, 3935-3943. 
20. Rissanou, A. N.; Georgilis, E.; Kasotakis, E.; Mitraki, A.; Harmandaris, V., Effect of solvent on the self-assembly of dialanine and diphenylalanine peptides. J. Phys. Chem. B 2013, 117, 3962-3975.

21. Guo, C.; Luo, Y.; Zhou, R.; Wei, G., Triphenylalanine peptides selfassemble into nanospheres and nanorods that are different from the nanovesicles and nanotubes formed by diphenylalanine peptides. Nanoscale 2014, 6, 2800-2811.

22. Frederix, P. W.; Ulijn, R. V.; Hunt, N. T.; Tuttle, T., Virtual screening for dipeptide aggregation: Toward predictive tools for peptide self-assembly. $J$. Phys. Chem. Lett. 2011, 2, 2380-2384.

23. Frederix, P. W.; Scott, G. G.; Abul-Haija, Y. M.; Kalafatovic, D.; Pappas, C. G.; Javid, N.; Hunt, N. T.; Ulijn, R. V.; Tuttle, T., Exploring the sequence space for (tri-)peptide self-assembly to design and discover new hydrogels. Nat. Chem. 2015, 7, 30-37.

24. Thota, N.; Ma, Y.; Jiang, J., Molecular insights into the self-assembly of short amphiphilic peptides $\mathrm{F}_{\mathrm{m}} \mathrm{D}_{\mathrm{n}}$ and $\mathrm{F}_{\mathrm{m}} \mathrm{K}_{\mathrm{n}}$. RSC $A d v$. 2014, 4, 6074160748

25. Emamyari, S.; Fazli, H., pH-dependent self-assembly of EAK16 peptides in the presence of a hydrophobic surface: Coarse-grained molecular dynamics simulation. Soft Matter 2014, 10, 4248-4257.

26. Thota, N.; Luo, Z.; Hu, Z.; Jiang, J., Self-assembly of amphiphilic peptide (AF) ${ }_{6} \mathrm{H}_{5} \mathrm{~K}_{15}$ : Coarse-grained molecular dynamics simulation. J. Phys. Chem. B 2013, 117, 9690-9698.

27. Thota, N.; Jiang, J., Self-assembly of amphiphilic peptide $(A F)_{6} \mathrm{H}_{5} \mathrm{~K}_{15}$ derivatives: Roles of hydrophilic and hydrophobic residues. J. Phys. Chem. B 2014, 118, 2683-2692.

28. Thota, N.; Hu, Z.; Jiang, J., Ibuprofen loading and release in amphiphilic peptide FA32 and its derivatives: A coarse-grained molecular dynamics simulation study. Mol. Simul. 2016, 42, 679-687.

29. Mondal, J.; Yethiraj, A., Effect of secondary structure on the self-assembly of amphiphilic molecules: A multiscale simulation study. J. Chem. Phys. 2012, 136, 084902.

30. Gudlur, S.; Sukthankar, P.; Gao, J.; Avila, L. A.; Hiromasa, Y.; Chen, J.; Iwamoto, T.; Tomich, J. M., Peptide nanovesicles formed by the selfassembly of branched amphiphilic peptides. PLoS One 2012, 7, e45374.

31. Lee, O. S.; Stupp, S. I.; Schatz, G. C., Atomistic molecular dynamics simulations of peptide amphiphile self-assembly into cylindrical nanofibers. J. Am. Chem. Soc. 2011, 133, 3677-3683.

32. Lee, O. S.; Liu, Y.; Schatz, G. C., Molecular dynamics simulation of $\beta$ sheet formation in self-assembled peptide amphiphile fibers. J. Nanopart. Res. 2012, 14, 936. 
33. Lee, O. S.; Cho, V.; Schatz, G. C., Modeling the self-assembly of peptide amphiphiles into fibers using coarse-grained molecular dynamics. Nano Lett. 2012, 12, 4907-4913.

34. Yu, T.; Schatz, G. C., Free-energy landscape for peptide amphiphile selfassembly: Stepwise versus continuous assembly mechanisms. J. Phys. Chem. B 2013, 117, 14059-14064.

35. Yu, T.; Schatz, G. C., Free energy profile and mechanism of self-assembly of peptide amphiphiles based on a collective assembly coordinate. J. Phys. Chem. B 2013, 117, 9004-9013.

36. Venkataraman, S.; Hedrick, J. L.; Ong, Z. Y.; Yang, C.; Ee, P. L.; Hammond, P. T.; Yang, Y. Y., The effects of polymeric nanostructure shape on drug delivery. Adv. Drug Deliv. Rev. 2011, 63, 1228-1246.

37. Márquez-Miranda, V.; Araya-Durán, I.; Camarada, M. B.; Comer, J.; Valencia-Gallegos, J. A.; González-Nilo, F. D., Self-assembly of amphiphilic dendrimers: The role of generation and alkyl chain length in siRNA interaction. Sci. Rep. 2016, 6, 29436.

38. Kasimova, A. O.; Pavan, G. M.; Danani, A.; Mondon, K.; Cristiani, A.; Scapozza, L.; Gurny, R.; Moller, M., Validation of a novel molecular dynamics simulation approach for lipophilic drug incorporation into polymer micelles. J. Phys. Chem. B 2012, 116, 4338-4345.

39. Patel, S.; Lavasanifar, A.; Choi, P., Application of molecular dynamics simulation to predict the compatability between water-insoluble drugs and self-associating poly(ethylene oxide)- $b$-poly( $\varepsilon$-caprolactone) block copolymers. Biomacromolecules 2008, 9, 3014-3023.

40. Patel, S. K.; Lavasanifar, A.; Choi, P., Roles of nonpolar and polar intermolecular interactions in the improvement of the drug loading capacity of PEO- $b$-PCL with increasing PCL content for two hydrophobic cucurbitacin drugs. Biomacromolecules 2009, 10, 2584-2591.

41. Patel, S. K.; Lavasanifar, A.; Choi, P., Molecular dynamics study of the encapsulation capability of a PCL-PEO based block copolymer for hydrophobic drugs with different spatial distributions of hydrogen bond donors and acceptors. Biomaterials 2010, 31, 1780-1786.

42. Razavilar, N.; Choi, P., Molecular dynamics study of the diffusivity of a hydrophobic drug cucurbitacin B in pseudo-poly(ethylene oxide- $b$ caprolactone) micelle environments. Langmuir 2014, 30, 7798-7803.

43. Costache, A. D.; Sheihet, L.; Zaveri, K.; Knight, D. D.; Kohn, J., Polymerdrug interactions in tyrosine-derived triblock copolymer nanospheres: A computational modeling approach. Mol. Pharm. 2009, 6, 1620-1627.

44. Guo, X. D.; Tan, J. P.; Kim, S. H.; Zhang, L. J.; Zhang, Y.; Hedrick, J. L.; Yang, Y. Y.; Qian, Y., Computational studies on self-assembled paclitaxel structures: Templates for hierarchical block copolymer assemblies and sustained drug release. Biomaterials 2009, 30, 6556-6563. 
45. Guo, X. D.; Qian, Y.; Zhang, C. Y.; Nie, S. Y.; Zhang, L. J., Can drug molecules diffuse into the core of micelles? Soft Matter 2012, 8, 9989-9995.

46. Nie, S. Y.; Sun, Y.; Lin, W. J.; Wu, W. S.; Guo, X. D.; Qian, Y.; Zhang, L. J., Dissipative particle dynamics studies of doxorubicin-loaded micelles assembled from four-arm star triblock polymers 4AS-PCL- $b$-PDEAEMA- $b$ PPEGMA and their pH-release mechanism. J. Phys. Chem. B 2013, 117, 13688-13697.

47. Lin, W.; Nie, S.; Xiong, D.; Guo, X.; Wang, J.; Zhang, L., pH-responsive micelles based on (PCL) $)_{2}$ (PDEA- $b$-PPEGMA $)_{2}$ miktoarm polymer: Controlled synthesis, characterization, and application as anticancer drug carrier. Nanoscale Res. Lett. 2014, 9, 243.

48. Lin, W. J.; Nie, S. Y.; Chen, Q.; Qian, Y.; Wen, X. F.; Zhang, L. J., Structure-property relationship of $\mathrm{pH}$-sensitive (PCL) 2 (PDEA- $b$ PPEGMA) 2 micelles: Experiment and DPD simulation. AIChE J. 2014, 60, 3634-3646.

49. Nie, S. Y.; Lin, W. J.; Yao, N.; Guo, X. D.; Zhang, L. J., Drug release from $\mathrm{pH}$-sensitive polymeric micelles with different drug distributions: Insight from coarse-grained simulations. ACS Appl. Mater. Interfaces 2014, 6, 17668-17678.

50. Posocco, P.; Fermeglia, M.; Pricl, S., Morphology prediction of block copolymers for drug delivery by mesoscale simulations. J. Mater. Chem. 2010, 20, 7742-7753.

51. Luo, Z.; Jiang, J., pH-sensitive drug loading/releasing in amphiphilic copolymer PAE-PEG: integrating molecular dynamics and dissipative particle dynamics simulations. J. Control. Release 2012, 162, 185-193.

52. Jiang, W.; Luo, J.; Nangia, S., Multiscale approach to investigate selfassembly of telodendrimer based nanocarriers for anticancer drug delivery. Langmuir 2015, 31, 4270-4280.

53. Loverde, S. M.; Klein, M. L.; Discher, D. E., Nanoparticle shape improves delivery: rational coarse grain molecular dynamics (rCG-MD) of taxol in worm-like PEG-PCL micelles. Adv. Mater. 2012, 24, 3823-3830.

54. Srinivas, G.; Mohan, R. V.; Kelkar, A. D., Polymer micelle assisted transport and delivery of model hydrophilic components inside a biological lipid vesicle: A coarse-grain simulation study. J. Phys. Chem. B 2013, 117, 12095-12104.

55. De Nicola, A.; Hezaveh, S.; Zhao, Y.; Kawakatsu, T.; Roccatano, D.; Milano, G., Micellar drug nanocarriers and biomembranes: How do they interact? Phys. Chem. Chem. Phys. 2014, 16, 5093-5105.

56. Tang, Y.-H.; Li, Z.; Li, X.; Deng, M.; Karniadakis, G. E., Non-equilibrium dynamics of vesicles and micelles by self-assembly of block copolymers with double thermoresponsivity. Macromolecules 2016, 49, 2895-2903.

57. Jämbeck, J. P. M.; Eriksson, E. S. E.; Laaksonen, A.; Lyubartsev, A. P.; Eriksson, L. A., Molecular dynamics studies of liposomes as carriers for 
photosensitizing drugs: Development, validation, and simulations with a coarse-crained model. J. Chem. Theory Comput. 2014, 10, 5-13.

58. Fu, I. W.; Markegard, C. B.; Chu, B. K.; Nguyen, H. D., The role of electrostatics and temperature on morphological transitions of hydrogel nanostructures self-assembled by peptide amphiphiles via molecular dynamics simulations. Adv. Healthc. Mater. 2013, 2, 1388-1400.

59. Fu, I. W.; Markegard, C. B.; Nguyen, H. D., Solvent effects on kinetic mechanisms of self-assembly by peptide amphiphiles via molecular dynamics simulations. Langmuir 2015, 31, 315-324.

60. Fu, I. W.; Markegard, C. B.; Chu, B. K.; Nguyen, H. D., Role of hydrophobicity on self-assembly by peptide amphiphiles via molecular dynamics simulations. Langmuir 2014, 30, 7745-7754.

61. Guo, X. D.; Zhang, L. J.; Wu, Z. M.; Qian, Y., Dissipative particle dynamics studies on microstructure of $\mathrm{pH}$-sensitive micelles for sustained drug delivery. Macromolecules 2010, 43, 7839-7844.

62. Guo, X. D.; Zhang, L. J.; Qian, Y., Systematic multiscale method for studying the structure-performance relationship of drug-delivery systems. Ind. Eng. Chem. Res. 2012, 51, 4719-4730.

63. Wang, Y.; Li, Q. Y.; Liu, X. B.; Zhang, C. Y.; Wu, Z. M.; Guo, X. D., Mesoscale simulations and experimental studies of $\mathrm{pH}$-sensitive micelles for controlled drug delivery. ACS Appl. Mater. Interfaces 2015, 7, 2559225600.

64. Chen, L.; Jiang, T.; Cai, C.; Wang, L.; Lin, J.; Cao, X., Polypeptide-based "smart" micelles for dual-drug delivery: A combination study of experiments and simulations. Adv. Healthc. Mater. 2014, 3, 1508-1517.

65. Shen, Z.; Loe, D. T.; Awino, J. K.; Kroger, M.; Rouge, J. L.; Li, Y., Selfassembly of core-polyethylene glycol-lipid shell (CPLS) nanoparticles and their potential as drug delivery vehicles. Nanoscale 2016, 8, 14821-14835. 